\title{
Post-Graduate Training and Professional Development: Exploring the Nexus Between Self Compassion and Compassion Fatigue Among Mental Health Clinicians Working With Trauma Survivors
}

\author{
Nicole L. Arkadie ${ }^{1} \&$ Allen E. Lipscomb ${ }^{2}$ \\ ${ }^{1}$ Education Department, College of Doctoral Studies, Grand Canyon University, USA \\ 2 Social Work Department, College of Social and Behavioral Sciences, California State University Northridge, \\ Northridge, USA \\ Correspondence: Allen E. Lipscomb, PsyD, MSW, LCSW, Assistant Professor, Social Work Department, College of \\ Social and Behavioral Sciences, California State University Northridge, 18111 Nordhoff St. Northridge, CA, 91330, \\ USA. Tel: (818)677-5945.
}

Received: November 4, 2018

Accepted: November 28, 2018

Online Published: December 2, 2018

doi:10.5430/irhe.v3n4p41

URL: https://doi.org/10.5430/irhe.v3n4p41

\begin{abstract}
Mental health clinicians who work with clients who have experienced severe trauma are at greater risk of developing compassion fatigue. Limited prior research investigated the relationship between self-compassion and compassion fatigue. The purpose of this quantitative correlational study was to assess the relationships of self-compassion and duration of professional service to compassion fatigue among licensed mental health clinicians who worked with clients that have experienced trauma in southern California. Two research questions asked whether self-compassion and duration of professional service were significantly related to compassion fatigue. The researcher collected primary data for the variables of interest via an online survey using two validated instruments, SCS-SF and ProQOL-Version 5. The study was conducted with a convenience sample of $(n=67)$ licensed mental health clinicians who resided in southern California. The results of non-parametric Kendall's tau-b correlations revealed a significant inverse correlation between self-compassion and compassion fatigue, $\tau \mathrm{b}=-0.273, p=.002$. The correlation between duration of professional service and compassion fatigue was nonsignificant, $\tau \mathrm{b}=-0.104, p=.299$. These results are vital and relevant to the field as they justify further research, training and professional development in this area, leading to the development of clinical interventions that are needed to mitigate compassion fatigue symptoms among this population.
\end{abstract}

Keywords: compassion fatigue, mental health, training, self-compassion

\section{Introduction}

Compassion fatigue is a major problem for mental health clinicians, especially for those who work with clients who have experienced trauma. The problem of compassion fatigue has been examined for over twenty years. However, the focus has been on examining the effects of compassion fatigue and not on how to alleviate its symptoms. Compassion fatigue limits a mental health clinician's ability to have empathy and respect for their clients and has a negative impact on client care (Drury et al., 2014; Negash \& Sahin, 2011). There is a need for mental health clinicians to learn how to become more resilient to their clients' traumatic self-disclosures (O'Brien \& Haaga, 2015). Various studies have examined the relationship between compassion fatigue and other psychological health outcome measures, such as stress, anxiety, and burnout (Craig \& Sprang, 2010; Drury et al., 2014; Lee et al., 2015). None have examined the relationship between self-compassion, and duration of professional service, and compassion fatigue among licensed mental health clinicians who worked with trauma clients.

The self-compassion construct has been examined for the past ten years. The trends within the research over the last five years have focused on connecting self-compassion with positive health outcomes (Beaumont et al., 2016; Finlay-Jones et al., 2015; Neff, 2015; Raab et al., 2015; Raab, 2014; Sirois et al., 2015). Both Finlay-Jones et al.'s (2015) and Raab's (2014) study demonstrated that self-compassion had a positive relationship with psychological well-being and that it fostered resilience to stress (Finlay-Jones et al., 2015; Raab, 2014). Only Beaumont et al.'s (2016) study examined the relationship between self-compassion and compassion fatigue. The participants were student midwives and not mental health clinicians (Beaumont et al., 2016). Beaumont et al.'s (2016) study was 
limited to student participants whereas; this study used licensed mental health clinicians. This study addressed the current gap in the literature by examining the relationship between self-compassion, and duration of professional service, and compassion fatigue among mental health clinicians who worked with clients that have experience trauma.

O'Brien and Haaga (2015) identified the necessity of future studies to focus on skills that could build resiliency among mental health clinicians to protect against compassion fatigue. These researchers focused on the relationship between empathetic accuracy and compassion fatigue, not on the self-compassion variable among licensed mental health clinicians (O'Brien \& Haaga, 2015). Self-compassion has been viewed as a resiliency intervention skill that could mitigate symptoms of compassion fatigue/stress (Raab, 2014). Missing from the literature were studies that examined compassion fatigue among licensed mental health clinicians who worked with clients who experienced trauma. This study built on existing research by exploring a different population group of licensed mental health clinicians who worked with trauma clients and resided in Southern California. Also, this study filled the gap in the literature by examining the relationship between self-compassion, and duration of professional service, and compassion fatigue among the proposed population group (Finlay-Jones et al., 2015).

Researchers O'Brien and Haaga (2015) conducted a study that examined compassion fatigue and empathetic accuracy among non-licensed psychology trainees and clinical psychology doctoral students. These researchers noted a need for quantifiable statistical research to be conducted that examined compassion fatigue and self-compassion skills, as existing studies used qualitative approaches (O’Brien \& Haaga, 2015). As O'Brien and Haaga's (2015) study focused on compassion fatigue and empathetic accuracy and not on self-compassion interventions, they recommended that future research be done that examined possible ways to mitigate compassion fatigue among mental health clinicians.

Researchers Finlay-Jones et al.'s (2015) study was aimed at adding to existing literature by examining self-compassion and stress among Australian psychology trainees and psychology students. Finlay-Jones et al.'s (2015) study was limited to the use of psychology students and non-licensed mental health clinicians as research participants. This research study used only licensed mental health clinicians as participants. It was through Finlay-Jones et al.'s (2015) findings that they noted a need for future research to be conducted that examined self-compassion and specific occupational health outcomes of compassion fatigue and burnout (Finlay-Jones et al., 2015). This current study used a different population of sample participants than was used in Finlay-Jones et al.'s (2015) study and it examined the relationship between self-compassion and duration of professional service and compassion fatigue. To fill the gap in research, there remained a need to study if self-compassion and duration of professional service were significantly related to compassion fatigue among licensed mental health clinicians who work with clients that have experienced trauma.

\subsection{Theoretical Framework}

Various theories and models have been used as foundations to study compassion fatigue and self-compassion in the field of behavioral health. Transactional stress, multi-dimensional model of regulation difficulties, compassion theory, theory of caring, psycho-social stress theory, and emotional intelligence have been used in prior research to study compassion fatigue and self-compassion (Adams et al., 2006; Finlay-Jones et al., 2015; Gustin \& Wagner, 2012; Thompson et al., 2014; Zeidner et al., 2013). The self-compassion model is centered around being compassionate towards self during difficult times, and the SCS-SF was developed out of this model. The self-compassion model provides a deeper understanding of the self-compassion variable examined in this study. It is the absence of these main components of being non-judgmental and kind towards self during difficult times, being aware of thoughts and feelings without trying to change them and accepting self and failures as part of the human condition that results in compassion fatigue (Neff, 2015). It was through the examination of the main components of the self-compassion model that it became evident it was the model to use to investigate the problem being studied because it looks at the three constructs of self-compassion.

\subsection{Self-compassion Model}

The self-compassion model was birthed out of the need to study and understand how an individual could exercise compassion towards oneself during difficult times. Through the Buddhists teachings and writings that expressed the importance for an individual to exercise compassion towards self, the model of self-compassion was developed (Neff \& Pommier, 2013). Self-compassion is compassion turned inwards (Neff \& Pommier, 2013; Neff, 2015). It is defined as being non-judgmental towards self during difficult times, treating oneself with kindness and understanding when suffering, being mindful, aware of one's thoughts and feelings without trying to fix them, and recognizing that failures are a part of the human experience (Finlay-Jones et al., 2015; Lopez et al., 2015). There are three major 
components that make up the constructs of self-compassion: treating self with kindness and understanding during difficult times, while being non-judgmental towards ones' inadequacies and failures; cultivating mindful awareness of one's own suffering without over-identifying with thoughts and feelings, and common humanity; recognizing that one's experiences and failures are a part of being human (Finlay-Jones et al., 2015; Neff, 2003; Sirois et al., 2015).

The self-compassion model is broken into three subsets with a positive and negative for each component. One construct is self-kindness and understanding towards oneself versus self-criticism and judgment (Neff, 2015; Neff, 2003; Raab, 2014). It is the ability of a mental health clinician to be kind towards themselves without being hard or judgmental to self when they perceived having failed at something. Another construct is common humanity versus isolation, being able to recognize that everyone makes mistakes and that one is not alone in their suffering and failings (Neff, 2015; Neff, 2003; Raab, 2014). The final construct is mindfulness, being aware of one's thoughts and feelings versus over-identifying with them in an exaggerated, negative manner (Neff, 2015; Neff, 2003; Raab, 2014).

This study added to the self-compassion theory by examining its relationship to compassion fatigue using a different population than those found in previous studies. This study sought to determine if mental health clinicians who worked with clients who experienced trauma could have self-compassion and compassion fatigue simultaneously or if a statistically significant relationship existed between the variables. The data was analyzed using the SCS-SF (Neff, 2015) and the ProQOL-Version 5 (Stamm, 2010) instruments to determine if licensed mental health clinicians who worked with trauma reported low levels of self-compassion and high risk of compassion fatigue or high levels of self-compassion with low to no risk of compassion fatigue. The self-compassion model was chosen for this research study because it provided the best support for the research. It allowed this researcher to examine the level of self-compassion among the target population.

The self-compassion model has been studied by researchers for the past couple of decades. There has been a growing need to understand better how exercising kindness towards oneself in a non-judgmental way while understanding self as part of the human experience, could improve psychological functioning (Neff \& Pommier, 2013; Olson \& Kemper, 2014). A positive correlation has been found between having high levels of self-compassion and compassion for others and reduced levels of compassion fatigue (Beaumont et al., 2016). It has been suggested that a strong connection exists between self-compassion and psychological health (Neff \& Pommier, 2013). If a relationship is found to exist between compassion fatigue and self-compassion, intervention techniques could be used within a mental health organization to help mitigate the symptoms of compassion fatigue.

\section{Literature Review}

The condition of compassion fatigue has been examined for several decades whereas; self-compassion is a more recent construct that has been studied within the past decade. Lee et al. (2015) noted an increase in recent years of interest to research the phenomenon of self-compassion and its relationship to mindfulness, particularly as an outcome intervention. Within the field of behavioral health, risk levels of compassion fatigue have been examined among mental health clinicians (Adams et al., 2006; Lee et al., 2015; O'Brien \& Haaga, 2015; Thompson et al., 2014). Similarly, the level of self-compassion has been studied among this population group (Finlay-Jones et al., 2015; Raab et al., 2015). The research on the two constructs has been focused on defining them and gaining a better understanding of their psychometric properties.

Compassion fatigue is a psychological condition that has a negative impact on a mental health clinician's well-being (Lee et al., 2015). It reduces a mental health clinician's capacity to exercise empathy towards their clients (Thieleman \& Cacciatore, 2014). Compassion fatigue is thought to be a state of detachment and isolation that is experienced by healthcare professionals due to continuous engagement with distressed trauma clients (Lee et al., 2015). Mental health clinicians who experience compassion fatigue are considered at a greater risk for making poor clinical decisions related to misdiagnosing and inadequate treatment planning compared those who do not experience symptoms of compassion fatigue (Bride et al., 2007).

Self-compassion is a technique that has been found to enhance one's psychological health and well-being (Finlay-Jones et al., 2015; Sirois et al., 2015). In their study, Sirois et al. (2015) found that participants who were self-compassionate demonstrated positive behaviors that promoted health such as healthy eating, exercise, stress management, and reduced risk for diseases. An individual can have positive well-being by exercising compassion towards self with kindness and having a deep awareness of one's suffering (Neff, 2015; Raab, 2014). Prior research has shown that self-compassion exercises have reduced cortisol levels, which are triggered by stress and high anxiety (Beaumont et al., 2016; Raab, 2014). Examples of self-compassion exercises include activities such as, writing a letter to self as if one were responding to a close friend who was suffering from the same situation, or changing the negative self-critical talk to positive self-talk (Neff, 2017). 
In Beaumont's et al. (2016) study they found that participants who viewed themselves unsympathetically were less compassionate towards self and that led to greater reports of compassion fatigue. It is the ability to be aware of one's thought and feelings while not being critical with self-judgment that prevents a mental health clinician from suffering the effects of compassion fatigue (Beaumont et al., 2016; Raab et al., 2015). This study drew upon prior research conducted on compassion fatigue and self-compassion to investigate the relationship between the two constructs.

Of the reviewed literature, the researchers have examined the relationship between compassion fatigue and other subtheme variables such as job satisfaction, compassion satisfaction, empathy accuracy, burnout, anxiety state and trait anxiety (Craig \& Sprang, 2010; Ivicic \& Motta, 2016; Lee et al., 2015; O'Brien \& Haaga, 2015; Sodeke-Gregson et al., 2013). Beaumont et al. (2016) conducted a quantitative study that examined the relationship between self-compassion, compassion fatigue, and well-being among student midwives. However, absent from the literature was an examination of the relationship between self-compassion, and duration of professional service, and compassion fatigue among mental health clinicians who worked with trauma clients.

This study examined the link between self-compassion and duration of professional years and compassion fatigue among licensed mental health clinicians who worked with trauma clients. It was difficult for those who experienced compassion fatigue to regulate and manage their emotions due to what Merriman called "ineffective self-soothing behaviors" (Merriman, 2015). The negative condition of compassion fatigue causes a mental health clinician to be in a constant state of tension and experience high levels of anxiety (Thompson et al., 2014). A mental health clinician who is numb and emotionally distant while in session is not demonstrating an ability to regulate their emotions; this could have a negative impact on their clinical work. Self-Compassion has been found to be an effective intervention technique that regulates emotions and offers ways to demonstrate self-soothing mannerisms (Beaumont et al., 2016; Merriman, 2015). It was through the review of existing literature on compassion fatigue and self-compassion that the gap in research was identified.

\section{Methodology}

\subsection{Research Methodology}

This study used a quantitative methodology with a non-experimental correlational design to investigate if a relationship existed between self-compassion, professional years of service, and compassion fatigue. This approach was chosen because quantitative research attempts to establish statistically significant relationships between variables, determine attitude, describe trends, analyze numerical data to explain, and predict or control a phenomenon (Gay et al., 2014). A quantitative methodology approach was chosen because it allowed this researcher to assess the relationship between the variables, self-compassion and compassion fatigue. This researcher analyzed collected interval data using two standardized survey questionnaires that have been used previously in other research studies conducted on self-compassion and compassion fatigue.

\subsection{Participants and Procedure}

The convenience sample surveyed for this study consisted of 67 licensed mental health clinicians who worked with clients who experienced trauma and resided in southern California. To obtain a descriptive summary of the characteristics of the sample, demographic data were collected, for the following variables: professional discipline, leadership role, duration of professional service, occupational status, past trauma, and gender.

This researcher targeted for recruitment all licensed mental health clinicians who resided in southern California via a written announcement (recruitment script) that was posted on three social media sites, LinkedIn, Facebook, and Psychology Today. The announcement asked for volunteer participants to take part in the study and included a URL link to the online survey web page administered by Qualtrics. Each volunteer participant needed to accept the informed consent form by simply clicking the "I CONSENT" button before beginning the survey. The survey was anonymous as it collected no personal identification information. The raw data file was exported from Qualtrics as an Excel file. For tracking purposes, each survey response was assigned a sequential number from 1-67.

\subsection{Measures and Instrumentation}

The data was collected for this study using the online survey method and a questionnaire that included two validated instruments and a brief demographic questionnaire created by the researcher. The data for the self-compassion variable were collected using the SCS-SF instrument (Raes et al., 2011). The instrument utilized a five-point interval rating scale to evaluate individual responses in the survey, and the interval score of the scale is calculated as the mean. The SCS-SF is a 12-item self-report questionnaire that measures self-compassion using a five-point interval rating scale that ranges from 1 (almost never) to 5 (almost always) (Neff, 2003; Raes et al., 2011). The instrument has three subscales that were not used in the analysis: mindfulness, kindness, and common humanity (Neff, 2003). 
The data for the duration of the professional service variable were collected through a demographic question. The data for the compassion fatigue variable were collected using the ProQOL-Version 5 instrument. The ProQOL-Version 5 (Stamm, 2010) is a 30-item self-report questionnaire that has an overall scale of professional quality of life, with three subscales, one of which is secondary traumatic stress. The ProQOL-Version 5 has three subscales that measure compassion satisfaction, burnout, and secondary traumatic stress. The secondary traumatic stress subscale was utilized in this study to measure compassion fatigue. The ProQOL-Version 5 measurement scale is a five-point ordinal rating scale ranging from 1 (never), 2 (rarely), 3 (sometimes), 4 (often), to 5 (very often) (Stamm, 2010). Based on author's instructions and prior use of the instrument, this researcher approximated the ordinal data to interval and computed the scores for the secondary traumatic stress (i.e., compassion fatigue) as sums of the corresponding item scores (Stamm, 2010; Stamm, 2012; Beaumont et al., 2016; Sodeke-Gregson et al., 2013). The secondary traumatic stress subscale has 10 items, each rated 1 to 5 , so the scale range is $10-50$ points (Stamm, 2012). For this study only, the researcher operationalized compassion fatigue as secondary traumatic stress and used this subscale of the ProQOL Version 5 to measure compassion fatigue.

Three ranges of the overall scores on the ProQOL-Version 5 of the secondary traumatic stress subscale can be interpreted as ordinal levels: A score of 22 or less indicates low secondary traumatic stress, a score between 23-41 indicates a medium level of secondary traumatic stress, and a score of 42 or more indicates a high level of secondary traumatic stress (Stamm, 2010). However, the researcher used in the analysis of the interval scores on secondary traumatic stress subscales of the ProQOL-Version 5 to maximize score variance. The researcher operationalized compassion fatigue variable as secondary traumatic stress and used in the analysis of the data corresponding to this subscale of the ProQOL-Version 5. The data collected with this instrument are approximated to the interval, and the scale and subscale scores are computed as sums of the corresponding item scores (Stamm, 2010).

\subsection{Recruitment and Data Collection}

This researcher started the data collection procedure after approval had been granted by Grand Canyon University Institutional Review Board (IRB). The data was collected for this study via an online survey. The survey questionnaire included two validated instruments (SCS-SF and ProQOL-Version 5) and a brief demographic questionnaire created by the researcher. The survey was administered online through Qualtrics.

The first page of the online survey was the informed consent form. An announcement was written and posted on three social media sites: LinkedIn, Facebook, and Psychology Today. The announcement included three screening questions to ensure that the participants met the selection criteria - 1) Are they a licensed mental health clinician? 2) Do they reside in southern California? 3) Do they currently work or have previously worked with trauma clients?

The announcement asked for volunteer participants to take part in the research study by completing the survey questionnaire online using Qualtrics. A brief synopsis of the study and its purpose was provided as well as a statement explaining the importance of anonymity. The participants were informed that their participation was voluntary and that they could stop at any time during the survey without incurring negative consequences. Participants who responded to the announcement and met the selection criteria were prompted to click on a link that took them directly to the online survey administered through Qualtrics. When they clicked on the link, the survey opened to the informed consent form. Participants then clicked on the "I CONSENT" button before they could start the actual survey. The participants' names were not recorded in the survey or any forms. For tracking and identification purposes, the cases in the raw data file (exported from Qualtrics as an excel spreadsheet) were numerically coded.

The completed survey consisted of three sections. The first section included six demographic questions that collected data for the following sample descriptors: professional discipline, duration of professional service, gender, leadership or non-leadership role, occupational status, and past traumatic experience. The duration of professional service was also used as a variable of interest in the correlation analysis. The second section measured compassion fatigue and included all 30 questions from the ProQOL-Version 5. There were 10 questions for each of the three subscales: compassion satisfaction, burnout, and secondary traumatic stress. The researcher used in the analysis only the data corresponding to the secondary traumatic stress subscale, as that measured compassion fatigue. The third section included 12 questions from the SCS-SF scale regarding self-compassion. The survey took approximately up to 10 minutes to complete and was completed in one sitting.

The survey was open for five weeks. After the survey had been active for three weeks, this researcher posted another announcement on the same three social media sites previously mentioned and kept the survey open for two additional weeks. This researcher was able to obtain 67 participants. Once the data collection was completed and the SCS-SF scale and the secondary traumatic stress subscale of the ProQOL-Version 5 were computed, the researcher computed 
the descriptive statistics, checked the assumptions for the planned correlation analysis, and performed the correlation analysis needed to test the hypotheses and answer the two research questions:

RQ1: Is there a statistically significant relationship between self-compassion and compassion fatigue among licensed mental health clinicians who worked with trauma clients?

$\mathbf{H 1} \mathbf{1}_{\mathbf{0}}$ : There is no statistically significant relationship between self-compassion and compassion fatigue among mental health clinicians who worked with trauma clients.

H1a: There is a statistically significant relationship between self-compassion and compassion fatigue among mental health clinicians who worked with trauma clients.

RQ2: Is there a statistically significant relationship between the duration of professional service and compassion fatigue among licensed mental health clinicians who worked with trauma clients?

$\mathbf{H} \mathbf{2}_{\mathbf{0}}$ : There is no statistically significant relationship between the duration of professional service and compassion fatigue among licensed mental health clinicians who worked with trauma clients.

H2a: There is a statistically significant relationship between the duration of professional service and compassion fatigue among licensed mental health clinicians who worked with trauma clients.

The research questions and hypothesis provided the framework for testing the relationships of the variables. To examine both research questions and corresponding hypotheses, an inferential statistical analysis was conducted. This researcher proposed to use parametric Pearson's correlation analysis to test the first research question because both variables involved were an interval, and non-parametric Spearman's correlation analysis to answer the second research question because the duration of service was an ordinal variable and compassion fatigue was the interval (Laerd Statistics, 2018).

\section{Results}

This study employed descriptive and inferential statistical analyses. A Kendall tau-b non-parametric correlation analysis was used to analyze the data, test the hypotheses, and answer the research questions. This was a change from the original analysis plan to use parametric Pearson's correlation analysis for the first research question and non-parametric Spearman's correlation analysis.

\subsection{Descriptive Findings}

Seventy-three participants accepted the informed consent and began the survey, only 67 completed the survey. Six participants did not fill out the survey questionnaire, and their records were discarded. Thus, the final convenience sample for this study was comprised of 67 participants. Forty-nine $(73.13 \%)$ of the participants were clinical social workers, $8(11.94 \%)$ were marriage and family therapists, seven $(10.45 \%)$ were psychologists and three $(4.48 \%)$ identified their professional discipline as Other. The majority of the participants-39 $(58.21 \%)$ — stated they were in a leadership role, $28(41.79 \%)$ were not in a leadership role, $36(53.73 \%)$ reported having worked in mental health for $11+$ years; $25(37.31 \%)$ reported having worked in the field for 5-10 years; five (7.46\%) reported having worked 2-4 years, and only one (1.49\%) reported having $0-1$ duration of professional service.

The overwhelming majority of the participants - 63 (94.03\%) worked full-time, and only four participants (5.97\%) reported working part-time hours. The majority of licensed mental health clinicians within this study-47 (70.15\%) reported having experienced past trauma, and $20(29.85 \%)$ reported not having experienced any past trauma. The majority of the participants - 57 (85.07\%) identified themselves as females, and ten (14.93\%) identified themselves as males (Table 2). These results were consistent with previous studies that examined compassion fatigue among mental health professionals, where most participants were women. In Ivicic and Motta's (2016) study, 75\% of the participants were females. In a study by Sodeke-Gregson et al. (2013), $72 \%$ of the participants were females. In O'Brien and Haaga's (2015) study, $71 \%$ of the participants were females. The Lee et al. (2015) study was conducted with a sample in which women represented 97.5\%. Beaumont et al. (2016) reported that all participants in their study were females. 
Table 1. Frequency table for professional discipline, leadership role, duration of professional service, occupational status, past trauma, and gender of participants

Variable

n

\section{Professional Discipline}

Clinical social worker

Marriage \& family therapist

Other

Psychologist

\section{Leadership Role}

No

Yes

\section{Duration of Professional Service}

$0-1$ years
$2-4$ years
$5-10$ years
11 years or more

Occupational Status

full-time

part-time

Past Trauma

no

yes

\section{Gender}

Female

Male

Note. The total sample size for the study was $N=67$.

\subsection{Descriptive Statistics for the Variables of Interest}

The SCS-SF has an overall scale of self-compassion with three subscales representing dimensions of self-compassion: mindfulness, kindness, and common humanity (Neff, 2003). This researcher used in the analysis only the overall self-compassion score. In the analysis, the researcher used the overall scores, not the three levels, in order to maximize variance for this variable. One participant did not answer any of the 12 questions on the self-compassion survey. The total sample for the self-compassion scale was comprised of 66 participants.

Self-Compassion Scale had an average mean score of $3.61\left(S D=0.63, \mathrm{SE}_{\mathrm{M}}=0.08\right)$, with a minimum score of 2.17 and a maximum score of 4.92. The distribution for this variable had skewness -.013 and kurtosis - 0.62 (Table 2). When skewness and kurtosis are below 1 in absolute value, the shape of the variable distribution can be considered approximately normal, close to the bell shape curve (Intellectus Statistics, 2017). The majority of the participants-38 (56.72\%) — scored on the SCS-SF in the high-level range; $25(37.21 \%)$ scored in the moderate level range; only three (4.48\%) scored in the low-level range for self-compassion. 
Table 2. Summary descriptive statistics table for self-compassion and compassion fatigue

\begin{tabular}{|c|c|c|c|c|c|c|}
\hline Variable & $M$ & $S D$ & Min & $\operatorname{Max}$ & Skewness & Kurtosis \\
\hline Self-compassion scale & 3.61 & 0.63 & 2.17 & 4.92 & -0.14 & -0.62 \\
\hline $\begin{array}{l}\text { Compassion fatigue scale / } \\
\text { Secondary traumatic stress } \\
\text { scale }\end{array}$ & 21.57 & 5.77 & 11.00 & 36.00 & 0.37 & -0.45 \\
\hline
\end{tabular}

Note. The total sample for the study was $N=67$.

\subsection{Compassion Fatigue}

The Pro-QOL-Version 5 consists of three scales, Compassion satisfaction, Burnout, and Secondary traumatic stress (Stamm, 2010). The instrument has ten questions for each scale, with a total possible score ranging from 10 to 50 (Lee et al., 2015). The developers of the ProQOL-Version 5 Scale required all 30 questions to be included when using the instrument; researchers were not allowed to omit or remove any scales from the survey (Stamm, 2010). As this researcher was only interested in examining the compassion fatigue levels of participants, the compassion satisfaction scale scores and the burnout scale scores were not analyzed within this study.

Compassion fatigue was operationalized in this study as secondary traumatic stress and measured using the secondary traumatic stress subscale of the Pro-QOL-Version 5 (Stamm, 2010). The secondary traumatic stress scale had a mean score of $21.57\left(S D=5.77, \mathrm{SE}_{\mathrm{M}}=0.70\right)$, a skewness score of .37 , and a kurtosis score of .45 (Table 2). The skewness and kurtosis scores were below 1 (absolute value), indicating that the shape of the distribution of that variable was approximately normal (close to the normal bell curve) (Intellectus Statistics, 2017; Westfall \& Henning, 2013).

On the Secondary traumatic stress subscale, a sum score of 22 or less was considered Low, between 23 to 41 was Average, and 42 or more was High (Stamm, 2010). Table 3 shows the distribution of the secondary traumatic stress data collected from this study sample across the three ranges. The majority of the participants in this study -40 $(59.70 \%)$-- scored within the low range for compassion fatigue / secondary traumatic stress.

Table 3. Compassion fatigue levels

\begin{tabular}{lccc}
\hline \multicolumn{1}{c}{ Variable } & Levels & $\boldsymbol{n}$ & $\boldsymbol{\%}$ \\
\hline \multirow{3}{*}{$\begin{array}{l}\text { Compassion fatigue scale / } \\
\text { Secondary traumatic stress scale }\end{array}$} & Under 22-Low & 40 & 59.70 \\
\cline { 2 - 4 } & 22 to 41-Ave & 27 & 40.30 \\
\cline { 2 - 4 } & over 41-High & 0 & 0.00 \\
\hline
\end{tabular}

\subsection{Reliability}

Reliability refers to trustworthiness and dependability, the degree to which a test consistently measures whatever it states it will measure (Gay et al., 2014). In previous studies, the SCS-SF has been tested for the approval of its internal consistency, reliability, and test-retest (Neff, 2003; Neff, 2017; Raes et al., 2011). Finlay-Jones et al. (2015) utilized the SFC-SF to measure the link between the variable, self-compassion, and stress among Australian psychologists and psychology students. These researchers found that the SCS-SF had strong internal consistency (Cronbach's alpha $\geq .86 ; 84$ ), convergent and discriminant validity (Finlay-Jones et al., 2015).

In this study, Cronbach's alpha coefficients were evaluated using the guidelines suggested by George and Mallery (2016) where greater than .9 is considered excellent, greater than .8 is good, greater than .7 is acceptable, greater than .6 is considered questionable, greater than .5 is poor, and less than or equal to .5 is unacceptable. The self-compassion Cronbach's alpha score of .87 computed on the data collected for this study indicated good reliability. The secondary traumatic stress subscale of the ProQOL-Version 5 instrument was used to measure compassion fatigue. Previous studies found the ProQOL-Version 5 to have good internal consistency reliability, test-retest reliability, and convergent and discriminant validity (Finlay-Jones et al., 2015; Lee et al., 2015; 
Sodeke-Gregson et al., 2013;). The secondary traumatic stress scale showed a Cronbach's alpha coefficient of .87, which indicated good reliability.

\subsection{Nexus between Self-compassion and Compassion Fatigue}

The first research question was: Is there a statistically significant relationship between self-compassion and compassion fatigue among licensed mental health clinicians who work with trauma clients? The null hypothesis tested was that there would not be a statistically significant relationship between self-compassion and compassion fatigue among licensed mental health clinicians who work with trauma clients. The alternative hypothesis stated that there would be a statistically significant relationship between self-compassion and compassion fatigue among licensed mental health clinicians who work with trauma clients. The self-compassion variable was measured by SCS-SF scale. The compassion fatigue variable was measured by the secondary traumatic stress subscale of the ProQOL-Version 5.

The results revealed a statistically significant but low negative correlation between self-compassion and compassion fatigue, $\tau_{b}=-0.273, p=.002$. The results findings indicated that as self-compassion increased, compassion fatigue decreased. This tendency is apparent in the scatterplot presented in Figure 1. This statistically significant correlation provided support for the alternative hypothesis stated in the first research question. The researcher rejected the null hypothesis and concluded that there is a statistically significant inverse relationship between self-compassion and compassion fatigue among licensed mental health clinicians who worked with trauma clients.

Table 4. Kendall Tau-b Correlation Matrix: Self-compassion, duration of professional service, and secondary traumatic stress scale (compassion fatigue)

\begin{tabular}{|c|c|c|c|c|}
\hline Variables & Statistics & Self-Compassion & $\begin{array}{c}\text { Duration of } \\
\text { Service }\end{array}$ & $\begin{array}{c}\text { Compassion } \\
\text { Fatigue }\end{array}$ \\
\hline \multirow[t]{3}{*}{ Self-Compassion } & $\tau_{b}$ & 1.000 & 0.111 & $-.273^{* * *}$ \\
\hline & Sig. (2-tailed) & & 0.268 & 0.002 \\
\hline & $\mathrm{N}$ & 66 & 66 & 66 \\
\hline \multirow{3}{*}{$\begin{array}{l}\text { Duration of } \\
\text { Service }\end{array}$} & $\tau_{b}$ & 0.111 & 1.000 & -0.104 \\
\hline & Sig. (2-tailed) & 0.268 & & 0.299 \\
\hline & $\mathrm{N}$ & 66 & 67 & 67 \\
\hline \multirow{3}{*}{$\begin{array}{l}\text { Compassion } \\
\text { Fatigue }\end{array}$} & $\tau_{b}$ & $-.273^{* *}$ & -0.104 & 1.000 \\
\hline & Sig. (2-tailed) & 0.002 & 0.299 & \\
\hline & $\mathrm{N}$ & 66 & 67 & 67 \\
\hline
\end{tabular}

**. Correlation is significant at the 0.01 level (2-tailed).

The majority of the participants ( $n=40 ; 70 \%$ of the sample) scored within the low range for compassion fatigue and the vast majority of the participants scored within the high range for self-compassion ( $n=38 ; 56.72 \%$ of the sample) or within the moderate range $(n=25 ; 37.21 \%)$. Figure 1 provides a macro picture of the significant inverse relationship between self-compassion and compassion fatigue (using levels/ranges instead of scores), showing that at both moderate and high levels of self-compassion, more participants reported low compassion fatigue than moderate compassion fatigue. 


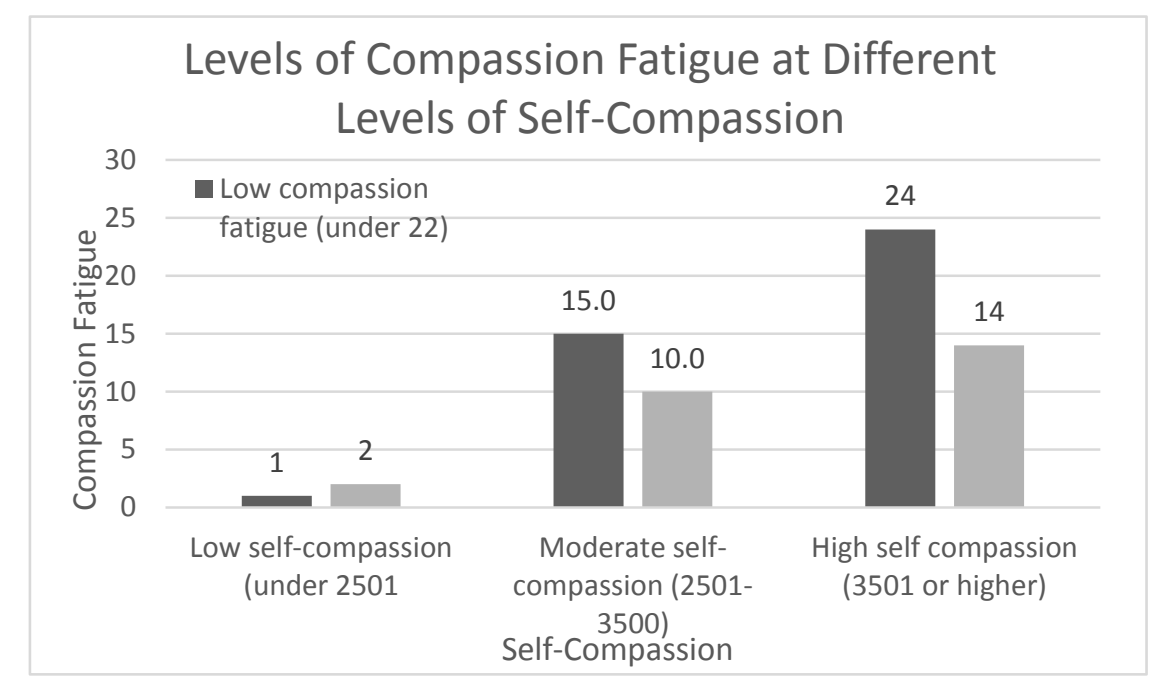

Figure 1. Bar chart of compassion fatigue levels at different levels of self-compassion

The second research question was: Is there a statistically significant relationship between the duration of professional service and compassion fatigue among licensed mental health clinicians who work with trauma clients? The null hypothesis stated that there would not be a statistically significant relationship that existed between the duration of professional service and compassion fatigue among licensed mental health clinicians who work with trauma clients. The alternative hypothesis stated that there would be a statistically significant relationship between the duration of professional service and compassion fatigue among licensed mental health clinicians who worked with trauma clients.

The result of Kendall's tau-b correlation analysis presented in indicated that there was not a statistically significant correlation between duration of professional service and compassion fatigue, $\tau_{b}=-0.104, p=.299$. The relationship was negative but weak, not reaching the level of statistical significance. Based on these findings, the researcher accepted the null hypothesis stated in the second research question.

\section{Discussion}

Results show that there is a statistically significant relationship between self-compassion and compassion fatigue among licensed mental health clinicians who work with clients who have exerpienced trauma. The analysis results revealed a statistically significant negative correlation between the self-compassion variable and the compassion fatigue variable, $\tau_{b}=-0.273, p=.002$. This result supported the alternative hypothesis stated in the first research question. The majority of the licensed mental health clinicians who participated in this study (close to 60\%) reported high levels of self-compassion and low levels of compassion fatigue. This finding may be used as a basis for using self-compassion as an intervention techniques to mitigate compassion fatigue among this population.

The results of this study were not consistent with the research on compassion fatigue that indicated mental health clinicians who worked with clients who have experienced trauma were at greater risk for developing compassion fatigue and prone to demonstrate signs of psychological distress (Adams et al., 2006; Craig \& Sprang, 2010; Figley, 2002; Negash \& Sahin, 2011; Newmeyer et al., 2016; O’Brien \& Haaga, 2015; Turgoose \& Maddox, 2017). All the licensed mental health clinicians who participated in this study reported having worked with clients with trauma, and most of them reported low levels of compassion fatigue. One of the screening criteria required the volunteer participants to have previously worked with trauma survivors. It is assumed that the participants in this study were truthful and met the screening criteria.

Also, it is unclear if the demographic characteristics of the licensed mental health clinicians had an impact on their reports of high levels of self-compassion and low levels of compassion fatigue. Most participants in this study were females $(85.07 \%)$; the majority of the participants were clinical social workers $(73.13 \%) ; 58.21 \%$ were in a leadership role; $53.73 \%$ had worked in the field of behavioral health for 11 or more years; $94.03 \%$ worked full-time; and $70.15 \%$ had experienced trauma. Craig and Sprang's (2010) study suggested that women were at a higher risk of developing compassion fatigue. Beaumont's et al. (2016) study found that women reported higher levels of compassion fatigue than their male counterparts. Most of the participants in this study were women (85.07\%), and 
most of them reported low levels of compassion fatigue (operationalized as secondary traumatic stress) (59.70\%).

The results of this study indicated that there was not a statistically significant relationship that existed between duration of professional service and compassion fatigue. Therefore, the null hypothesis that there was no statistically significant relationship between professional years of service and compassion fatigue among licensed mental health clinicians who worked with clients who experienced trauma was accepted, and the hypothesis was rejected.

The results of this study were not consistent with results of a study by Sodeke-Gregson et al. (2013) who found the length of time a mental health clinician worked in the field was a negative contributing factor of compassion fatigue: the fewer years a mental health clinician worked in the field, the higher the levels of compassion fatigue. The results of this current study were consistent with the findings of Lee et al. (2015) who reported no statistically significant relationship between compassion fatigue and years of professional experience among genetic counselors.

\subsection{Limitations}

Within research, limitations are aspects of the study that the researcher cannot control and may negatively affect the results and/or the generalizability of the results (Gay et al., 2014). A limitation was the use of a convenience sample. The target population of licensed mental health clinicians was very large $(82,090)$ within the state of California. The small convenience sample was chosen due to time constraints and its convenience. The volunteers were a very small subset of the target population. Additional concerns about sample representativeness and the generalizability of findings could arise in relation to the recruitment procedure. The researcher posted invitations on a small number of social media: Psychology Today, Facebook and LinkedIn. Those who do not frequently visit the social media sites may have missed the opportunity to participant in the study. Lastly, the data collection via online survey may have introduced self-reporting and/or desirability biases. Participants may have exaggerated their levels of self-compassion or under-reported their experience. The participants may have also embellished their compassion fatigue responses or under-reported it. Therefore, self-report research studies may have a problem with validity.

\section{Recommendations and Implications}

The results of this study provided significant findings that have theoretical implications as well as practical and future implications. The findings showed a negative statistically significant inverse relationship between self-compassion and compassion fatigue. The relationship between duration of professional service and compassion fatigue was inverse too, but weak and statistically nonsignificant. However, this study provided new data in an area that was limited in research that examined the presented variables. No earlier study had investigated the relationship between self-compassion and compassion fatigue among licensed mental health clinicians who worked with trauma survivors. The self-compassion model provided the theoretical framework for this study, and there were theoretical implications derived from the analysis findings. In addition, the results of this study can be applied for future training practice within a behavioral health setting. These results can be used to assist in the development of interventions to combat compassion fatigue. Also, the results of this study showed a link between self-compassion and compassion fatigue and this relationship should be further studied. An inverse relationship was found to exist between self-compassion and compassion fatigue. It is recommended that organizational and mental health leaders be trained to implement intervention programs that are designed to enhance self-compassion levels by promoting the use of self-compassion techniques. Implementing these types of training intervention programs could reduce mental health clinicians who work with trauma clients' chances of developing compassion fatigue. Organizational leaders placing emphasis on the importance of mental health clinicians' well-being, would demonstrate their commitment to the enhancement of employees' mental wellness.

\section{Conclusion}

Mental health professionals would benefit from reading the results of this study for their own professional development and training. These results provide empirical research information that mental health clinicians could use for educational purposes to mitigate compassion fatigue symptoms. Additionally, it is recommended that mental health clinicians be trained and encouraged to adopt daily self-care practices that incorporate self-compassion techniques. Ongoing practice and training to use self-compassion as an intervention technique would increase self-compassion levels and thus, reduce the levels of compassion fatigue among mental health clinicians. In addition, the authors believe that self-compassion as a daily technique could enhance mental health clinicians' work performance, improve their well-being, and increase their job satisfaction.

Furthermore, leadership within various practice settings could develop training and workshops to be presented to staff that promote the use of self-compassion techniques as a method to combat compassion fatigue. They could also incorporate these findings into their clinical supervision of staff, to encourage the use of self-compassion techniques. 


\section{References}

Adams, R., Boscarino, J., \& Figley, C. (2006). Compassion fatigue and psychological distress among social workers: A validation study. American Journal of Orthopsychiatry, 76(1), 103-108. https://doi.org/10.1037/0002-9432.76.1.103

Beaumont, E., Durkin, M., Martin, C. J. H., \& Carson, J. (2016). Compassion for others, self-compassion, quality of life and mental well-being measures and their association with compassion fatigue and burnout in student midwives: A quantitative survey. Midwifer, 34, 239-244. https://doi.org/10.1016/j.midw.2015.11.002

Boellinghaus, I., Jones, F. W., \& Hutton, J. (2013). Cultivating self-care and compassion in psychological therapists in training: The experience of practicing loving-kindness meditation. Journal of Training and Education in Professional Psychology, 7(4), 267-277. https://doi.org/10.1037/a0033092

Bride, B., Radey, M., \& Figley, C. (2007). Measuring compassion fatigue. Clinical Social Work Journal, 35, $155-163$. https://doi.org/10.1007/s10615-007-0091-7

California Board of Behavioral Science. (2016a). Licensed Psychologists 2016 report. Retrieved from www.psychology.ca.gov/index.shtml

California Board of Behavioral Science. (2016b). License and Registrant Statistics. Retrieved from www.bbs.ca.gov/app-reg/licereg_stats.shtml

Craig, C. D., \& Sprang, G. (2010). Compassion satisfaction fatigue, and burnout in a national sample of trauma treatment therapies. Anxiety, Stress \& Coping Journal, 23(3), 319-339. https://doi.org/10.1080/10615800903085818

Drury, V., Craigie, M., Francis, K., Aoun, S., \& Hegney, D. (2014). Compassion satisfaction, compassion fatigue, anxiety, depression and stress in registered nurses in Australia: Phase 2 results. The Journal of Nursing Management, 22, 519-531. https://doi.org/10.1111/jonm.12168

Figley, C. R. (2002). Compassion fatigue: Psychotherapists' chronic lack of self-care [SUP 1]. Journal of Clinical Psychology, 58(11), 1433-1441. https://doi.org/10.1002/jclp.10090

Finlay-Jones, A., Rees, C., \& Kane, R. (2015). Self-Compassion, emotion regulation and stress among Australian psychologists: Testing an emotion regulation model of self-compassion using structural equation modeling. PLoS ONE, 10(7), 1-19. https://doi.org/10.1371/journal.pone.0133481

Fulton, C. (2016). Mindfulness, self-compassion, and counselor characteristics and session variables. The Journal of Mental Health Counseling, 38(4), 360-374. https://doi.org/10.17744/mehc.38.4.06

Gay, L., Mills, G., \& Airasian, P. (2014). Educational research competencies for analysis and application (10th ed.). Dorling Kindersley, India: Pearson Educational Ltd.

George, D., \& Mallery, P. (2016). IBM SPSS statistics 23 step by step: A simple guide and reference. Routledge. https://doi.org/10.4324/9781315545899

Gustin, L. W., \& Wagner, L. (2012). The butterfly effect of caring-clinical nursing teachers' understanding of self-compassion as a source to compassionate care. Scandinavian Journal of Caring Sciences, 27, 175-183. https://doi.org/10.1111/j.1471-6712.2012.01033.x

Ingham-Broomfield, R. (2014). A nurses' guide to quantitative research. Australian Journal of Advanced Nursing, 32(2), 32-38.

Intellectus Statistics [Online computer software]. (2017). Retrieved from https://analyze.intellectusstatistics.com/

Ivicic, R., \& Motta, R. (2016). Variables associated with secondary traumatic stress among mental health professionals. Traumatology, 1-9. https://doi.org/10.1037/trm0000065

Kemper, K., Mo, X., \& Khayat, R. (2015). Are mindfulness and self-compassion associated with sleep and resilience in health professionals?. The Journal of Alternative and Complementary Medicine, 21(8), 496-503. https://doi.org/10.1089/acm.2014.0281

Laerd Statistics. (2018). Basic Requirements of Kendall's tau-b. Retrieved from https://statistics.laerd.com/premium/spss/ktb/kendalls-tau-b-in-spss-3.php

Lee, J. E. C., Sudom, K. A., \& Zamorski, M. A. (2013). Longitudinal analysis of psychological resilience and mental health in Canadian military personnel returning from overseas deployment. Journal of Occupational Health 
Psychology, 18(3), 327-337. https://doi.org/10.1037/a0033059

Lee, W., Veach, P., MacFarlane, I., \& Leroy, B. (2015). Who is at risk for compassion fatigue? An investigation of genetic counselor demographics, anxiety, compassion satisfaction, and burnout. National Society of Genetic Counselors, 24, 358-367. https://doi.org/10.1007/s10897-014-9716-5

Lopez, A., Sanderman, R., Smink, A., Zhang, Y., Sonderen, E. V., Ranchor, A., \& Schroevers, M. J. (2015). A reconsideration of the self-compassion subscale's total score: Self-compassion versus self-criticism. Plos One, 10(7), 1-12. https://doi.org/10.1371/journal.pone.0132940

Merriman, J. (2015). Enhancing counselor supervision through compassion fatigue education. Journal of Counseling \& Development, 93, 367-378. https://doi.org/10.1002/jcad.12035

Neff, K. (2017). Self-compassion guided meditations and exercises. Retrieved from http://self-compassion.org/category/exercises/\#exercises

Neff, K. D. (2003). The development and validation of a scale to measure self-compassion. Self \& Identity, 2(3), 223. https://doi.org/10.1080/15298860309027

Neff, K. D. (2015). The self-compassion subscale is a valid and theoretically coherent measure of self-compassion. Mindfulness, 7, 264-274. https://doi.org/10.1007/s12671-015-0479-3

Neff, K., \& Pommier, E. (2013). The relationship between self-compassion and other-focused concern among college undergraduates, community adults, and practicing mediators. Self and Identity, 12, 160-176. https://doi.org/10.1080/15298868.2011.649546

Negash, S., \& Sahin, S. (2011). Compassion fatigue in marriage and family therapy: Implications for therapists and clients. Journal of Marital and Family Therapy, 37(1), 1-13. https://doi.org/10.1111/j.1752-0606.2009.00147.x

Newmeyer, M., Keyes, B., Palmer, K., Kent, V., Spong, S., Stephen, F., \& Troy, M. (2016). Spirituality and religion as mitigating factors in compassion fatigue among trauma therapists in Romania. Journal of Psychology \& Theology, 44(2), 142-151. https://doi.org/10.1177/009164711604400205

O'Brien, J., \& Haaga, D. A. F. (2015). Empathetic accuracy and compassion fatigue among therapist trainees. Professional Psychology: Research and Practice, 46(6), 414. https://doi.org/10.1037/pro0000037

Olson, L., \& Kemper, K. J. (2014). Factors associated with well-being and confidence in providing compassionate care. Journal of Evidence-Based Complementary \& Alternative Medicine, 19(4), 292-296. https://doi.org/10.1177/2156587214539977

Patsiopoulos, A. T., \& Buchanan, M. J. (2011). The practice of self-compassion in counseling: A narrative inquiry. Professional Psychology: Research and Practice, 42(4), 301. https://doi.org/10.1037/a0024482

Raab, K. (2014). Mindfulness, self-compassion, and empathy among health care professionals: A review of the literature. Journal of Health Care Chaplaincy, 20, 95-108. https://doi.org/10.1080/08854726.2014.913876

Raab, K., Sogge, K., Parker, N., \& Flament, M. (2015). Mindfulness-based stress reduction and self-compassion among mental health professionals: a pilot study. The Journal of Mental Health, Religion, \& Culture 18(6), 503-512. https://doi.org/10.1080/13674676.2015.1081588

Radey, M., \& Figley, C. (2007). The social psychology of compassion. Clinical Social Work Journal, 35, 207-214. https://doi.org/10.1007/s10615-007-0087-3

Raes, F., Pommier, E., Neff, K. D., \& Van, G. (2011). Construction and factorial validation of short form of the self-compassion subscale. Clinical Psychology \& Psychotherapy, 18, 250-255. https://doi.org/10.1002/cpp.702

Shaughnessy, J. J., Zechmeister, E. B., \& Zechmeister, J. S. (2012). Research methods in psychology (9th ed.). New York, NY: McGraw-Hill.

Sirois, F., Hirsch, J., \& Kitner, R. (2015). Self-compassion, affect, and health-promoting behaviors. Journal of Health Psychology, 34(6), 661-669. https://doi.org/10.1037/hea0000158

Sodeke-Gregson, E. A., Holttum, S., \& Billings, J. (2013). Compassion satisfaction, burnout, and secondary traumatic stress in UK therapists who work with adult trauma clients.

Stamm, H. B. (2010). The Concise ProQOL Manual (2nd ed.). Pocatello, ID: ProQOL.org.

Thieleman, K., \& Cacciatore, J. (2014). Witness to suffering: Mindfulness and compassion fatigue among traumatic volunteers and professionals. Journal of Social Work, 59(1), 34-41. https://doi.org/10.1093/sw/swt044 
Thompson, I. A., Amatea, E. S., \& Thompson, E. S. (2014). Personal and contextual predictors of mental health counselors' compassion fatigue and burnout. Journal of Mental Health Counseling, 36(1), 58-77. https://doi.org/10.17744/mehc.36.1.p61m73373m4617r3

Westfall, P. H., \& Henning, K. S. S. (2013). Texts in statistical science: Understanding advanced statistical methods. Boca Raton, FL: Taylor \& Francis.

Zeidner, M., Hadar, D., Matthews, G., \& Roberts, R. (2013). Personal factors related to compassion fatigue in mental health professionals. Journal of Anxiety, Stress \& Coping, 26(6), 595-609. https://doi.org/10.1080/10615806.2013.777045

Zessin, U., Dickhauser, O., \& Garbade, S. (2015). The relationship between self-compassion and well-being: A meta-analysis. The International Association of Applied Psychology, 7(3), 340-364. https://doi.org/10.1111/aphw.12051 